\title{
Die Verwendung des Ultra-Turrax zur Aufteilung von Bakterienaggregaten in marinen Proben ${ }^{1}$
}

\author{
WILFRIED GUNKEL \\ Biologische Anstalt Helgoland, Meeresstation, Helgoland
}

\begin{abstract}
The use of the Ultra-Turrax for separation of bacterial aggregates in marine samples. Marine samples were treated with a homogenizer, Type Ultra-Turrax TP 18/2, 24,000 rpm. (Janke \& Kunkel, Staufen im Breisgau). In shore sediments rich in organic material, treatment with the Ultra-Turrax resulted in an increase in bacterial numbers (pourplate method) up to 119 times, compared with the results obtained from an untreated sample. The Ultra-Turrax has proven to be a useful tool, separating bacterial aggregates by mechanical effects. In sediment samples taken several miles offshore, there was a constant but only slight increase in the bacterial numbers up to $237 \%$. Experiments on sea water samples treated with the homogenizer showed varying results, with values occasionally slightly above or slightly below those of the untreated samples.
\end{abstract}

\section{EINLEITUNG}

Untersuchungen über die Rolle der Bakterien in der Okonomie des Meeres benötigen praktisch stets eine Feststellung ihrer Anzahl (BuCK \& CLEVERDoN 1961). Fast ausschließlich werden hierzu Kulturverfahren, etwa das Gußplattenverfahren, das Membranfilterkulturverfahren oder die Verdünnungsmethode (MPN-Methode) verwandt. In allen Fällen wird hier die Propagation eines Bakteriums, die entweder in der Bildung einer Kolonie oder der Trübung einer Nährlösung resultiert, gewertet. Bei diesen Verfahren können nur völlig voneinander getrennte Bakterien als verschieden erkannt werden: Der positiven Reaktion kann nicht entnommen werden, ob die Ursache ein oder mehrere - noch in irgendeinem Konnex stehende - Bakterien waren. Neben räumlich vereinzelt vorkommenden Bakterien finden wir im natürlichen Milieu jedoch stets Formen, die miteinander Aggregate bilden oder in großer Auswahl auf winzigen organischen oder anorganischen Partikeln als Aufwuchs erscheinen.

Eine Aufteilung der Bakterien in den verschiedenen Proben ist bisher mit mehr oder weniger großem Erfolg auf verschiedene Weise versucht worden, etwa durch starkes Schütteln, Schütteln mit Glasperlen, Einsatz von Magnetrührern sowie elektrischen Mixgeräten.

JONES \& JANNASCH (1959) untersuchten, ob eine Aufteilung der Bakterienklumpen des Seewassers mit Hilfe oberflächenaktiver Stoffe möglich ist. Sie fanden in ihren

\footnotetext{
1 Herrn Professor Dr. Adolf Bückmann zum 65. Geburtstag in Verehrung gewidmet.
} 
Experimenten einen Anstieg der mit der Gußplattenmethode erhaltenen Zahlen auf durchschnittlich fast das Doppelte gegenüber unbehandelten Kontrollen, stellten jedoch mit steigender Konzentration der oberflächenaktiven Stoffe eine Abnahme der Anzahl verschiedener Kolonietypen fest. Sie kamen zu dem Schluß, daß hier hauptsächlich resistente Formen verteilt werden, während für einen Teil der Bakterien die oberflächenaktiven Stoffe toxisch wirken. Abgesehen hiervon konnten Buck \& CLEvERdon (1961) die Ergebnisse von JoNEs \& JANNASCH an Seewasserproben von der Küste Connecticuts nicht bestätigen.

THoN berichtete über Versuche zur Feststellung der Gesamtkeimzahl in Nahrungsund Futtermitteln. Er verglich Bakterienzahlen, die beispielsweise aus Fischmehl nach verschiedener Vorbehandlung erhalten wurden. Pro Gramm Fischmehl erhielt THON nach 15 Minuten Handmörserung 11000 Bakterienkolonien; im elektrischen Mixer ethielt er nach 5 Minuten Einwirkungszeit 25000 und nach 2 Minuten Homogenisation mit Hilfe des Ultra-Turrax 780000 Bakterienkolonien. Die vorhandenen Bakterienaggregate wurden also mit Hilfe des Ultra-Turrax weitgehend aufgeteilt.

Im Nachfolgenden wird über Untersuchungen berichtet, in denen der UltraTurrax zur Aufteilung von Bakterienaggregaten in marinen Proben verwandt wurde.

\section{METHODIK}

Das Gerät: Es wurde ein Ultra-Turrax, Typ 18/2, der Firma Janke \& Kunkel, Staufen i. Br., verwandt. Es handelt sich hier um ein mechanisches Hochfrequenzgerät, das bis zu $24000 \mathrm{U} / \mathrm{min}$ leistet. Durch Hervorrufung von Druck, Beschleunigungsgefälle, Stoß, Prall, Kavitation und Scherung in den zu untersuchenden Medien stellt es einen vorzüglichen Homogenisator dar (Wrizms 1956, Fuchs 1960).

Das Gerät besteht aus einem Motorteil und einem abschraubbaren Schaft von $165 \mathrm{~mm}$ Länge und $18 \mathrm{~mm}$ Durchmesser aus säurebeständigem Stahl von V2A/V4AQualität. Der Schaft kann laut Angabe der Herstellerfirma sowohl durch kurzen Lauf des Gerätes in 70\% \% Alkohol als auch in einem anderen desinfizierenden Mittel sterilisiert werden. Es ist aber auch möglich, ihn im Autoklaven zu sterilisieren, wobei die Temperatur jedoch nicht über $120^{\circ} \mathrm{C}$ betragen sollte.

Das Untersuchungsmaterial: Es stammte sämtlich aus dem Gebiet um Helgoland. Das zu den Versuchen verwandte Seewasser wurde mit Hilfe des J. Z.Samplers (ZoBelL 1946) unter Einhaltung steriler Cautelen an der zwischen der Insel Helgoland und der Düne gelegenen Kabeltonne beziehungsweise der Tonne N/A entnommen. Hier herrscht ständig ein starker Gezeitenstrom, der sich mit ab- und auflaufendem Wasser umkehrt. Ein stärkerer Inseleinfluß besteht nicht, so daß fast ausschließlich rein marine Bakterien vorhanden sein dürten. Die Proben wurden umgehend nach der Entnahme in das Labor gebracht, so daß bis zum Beginn der Versuche nie mehr als 15 Minuten und bis zu deren Beendigung nie mehr als 2 Stunden vergingen.

Küstennahe Sedimente wurden am Helgoländer Strand oder hinter der Westmauer entnommen. Diese Sedimente waren in allen Fällen reich an organischer Substanz, hatten ein negatives Redoxpotential und waren durch Bildung von Hydrotroilit schwarz gefärbt. Küstenfernere Sedimente wurden auf dem Hever-Schnitt mit dem 
van Veen-Bodengreifer von Bord des Forschungskutters "Uthörn" entnommen und sofort an Bord aufgearbeitet.

Eine mit dem Planktonnetz gefischte Planktonprobe, die fast ausschließlich aus Ceratiumarten bestand, wurde aufgearbeitet, nachdem sie mehrere Tage im Labor gestanden hatte und das Plankton weitgehend abgestorben war. Von einem tiefgefrorenen Hering wurde ein Stück Fleisch ohne Haut und Gräten mit dem UltraTurrax aufgearbeitet. $\mathrm{pH}$ - und Eh-Messungen erfolgten mit dem Beckmann-pHMeter G (Batteriegerät) sofort nach der Entnahme.

Durchführung der Homogenisation: Seewasserproben von jeweils $200 \mathrm{ml}$ wurden in einem Becherglas direkt homogenisiert; bei der Untersuchung von

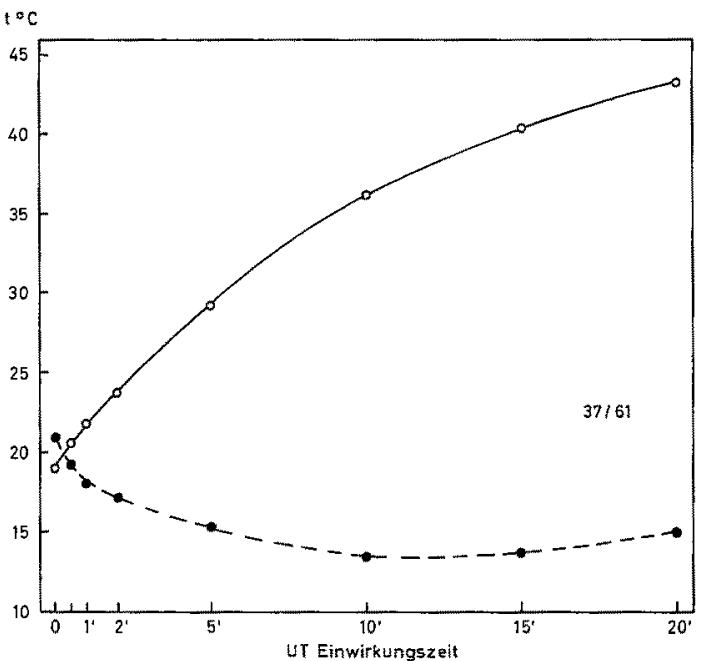

Abb. 1: Temperaturverlauf in einer Suspension von $20 \mathrm{~g}$ Sediment in $200 \mathrm{ml}$ Seewasser während der Einwirkungszeit des Ultra-Turrax. $A$ Ohne Kühlung; $B$ Kühlung durch ein Eis-Wassergemisch

Sedimenten wurden $2 \mathrm{~g}$ Material in $200 \mathrm{ml}$ steriles Seewasser in ein Becherglas eingebracht. $D a$ die meisten Meeresbakterien sehr temperatursensitiv sind und bereits bei kurzzeitiger Einwirkung von Temperaturen oberhalb $30^{\circ} \mathrm{C}$ abgetötet werden (ZoBELL 1946), war es notwendig, den Temperaturverlauf in der Suspension während des Laufes des Gerätes, der mehrere Minuten betrug, zu überwachen. Die Temperatur stieg nach 10 Minuten Laufzeit von $19^{\circ}$ auf $36^{\circ} \mathrm{C}$ und nach 20 Minuten sogar auf $43^{\circ} \mathrm{C}$. Wurde das Becherglas mit der Probe während des Gerätelaufes in einen Behälter mit Eiswasser gestellt, fand keine Temperaturerhöhung statt, sondern die Temperatur lag nach 20 Minuten Laufzeit sogar unter dem Anfangswert (Abb. 1). Die Temperatur wurde mit einem einfachen Quecksilber-Thermometer gemessen, zur Messung wurde das Gerät kurz abgeschaltet. Die Dauer der Abschaltung entsprach derjenigen bei der Entnahme zur Ausplattung und betrug nicht mehr als 1 Minute. Sämtliche nachfolgend angeführten Untersuchungen wurden aus den genannten Gründen unter Kühlung mit Eiswasser durchgeführt. Die Homogenisation sandiger Sedimente führt nach Auf- 
arbeitung einer größeren Anzahl Proben zu Abnutzungserscheinungen am distalen Ende des Schaftes. Dieser kann jedoch leicht und ohne großen Aufwand ersetzt werden.

Die Keimzählung: Nach verschiedenen Laufzeiten des Gerätes wurden Proben entnommen, Verdünnungsreihen durchgefuhrt und unter Verwendung des bekannten Seewasseragarmediums 2216 E Gußplatten angelegt. Nach 21 Tagen Bebrütung bei $18^{\circ} \mathrm{C}$ wurde die Anzahl gebildeter Kolonien mit Hilfe eines Zählpultes mit indirekter Beleuchtung und Vergrößerungsglas gezählt.

\section{ERGEBNISSE UND DISKUSSION}

Die Abbildungen 2 bis 6 zeigen den Einfluß der Homogenisation mit Hilfe des Ultra-Turrax auf die Bakterienzahl von Proben marinen Ursprungs. Die Darstellung ist doppelt linear, auf der Ordinate ist die Anzahl Bakterien pro ml Ausgangssuspen-

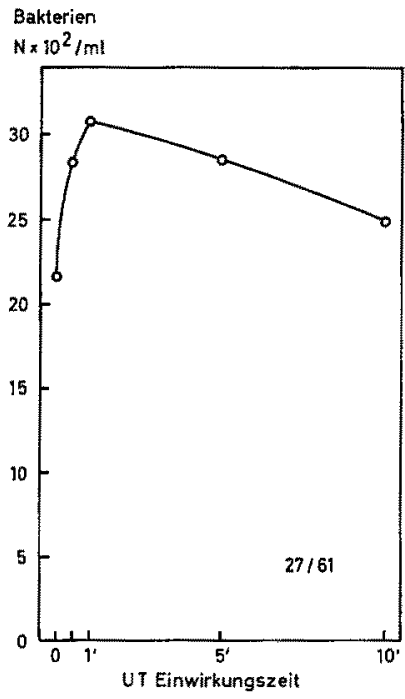

Abb. 2

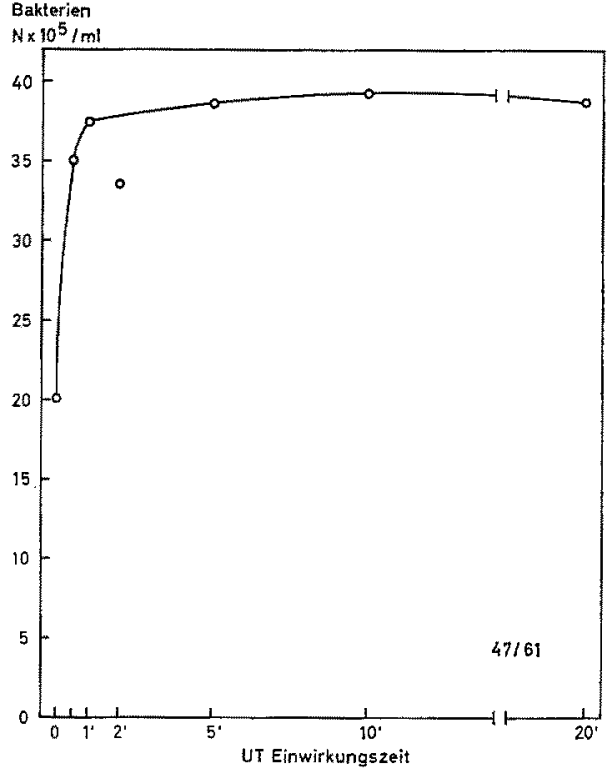

Abb. 3

Abb. 2: Einfluß der Homogenisation auf die Bakterienzahl von Seewasser Abb. 3: Einfluß der Homogenisation auf die Bakterienzahl einer Probe abgestorbenen Ceratienplanktons

sion, pro g Trockengewicht beziehungsweise pro g Feuchtgewicht aufgetragen, auf die Abszisse die Laufzeit des Gerätes.

In der Seewasserprobe wird nur eine geringfügige Vermehrung erreicht, nach kurzer Laufzeit tritt bereits wieder eine Abnahme ein. Weitere Versuche mit Seewasser wiesen unregelmäßige Ergebnisse auf, und zu einem beträchtlichen Teil lagen die Werte nach Behandlung unter dem Anfangswert (siehe auch Abb. 7). Hier muß mit 


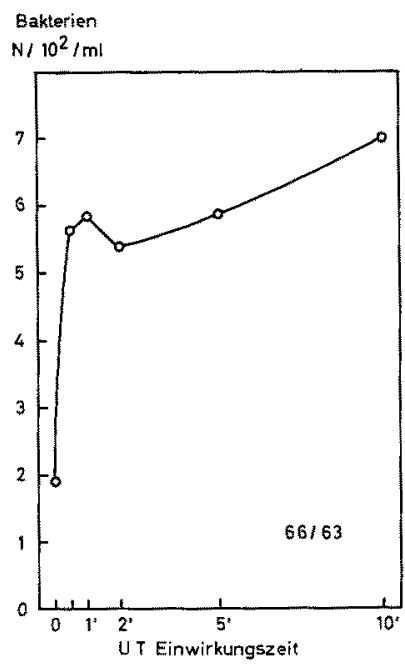

Abb. 4

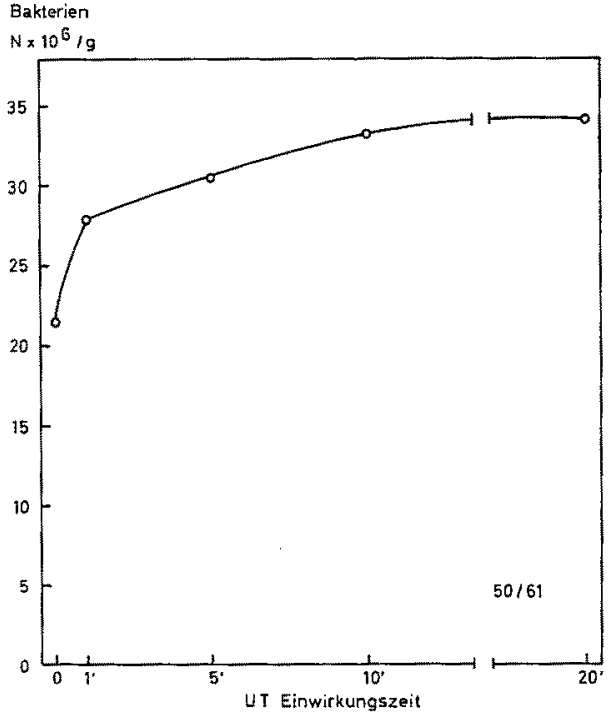

Abb. 5

Abb. 4: Einfluß der Homogenisation auf die Bakterienzahl einer Fischfleischprobe Abb. 5: Einfluß der Homogenisation auf die Bakterienzahl eines küstenfernen Sediments

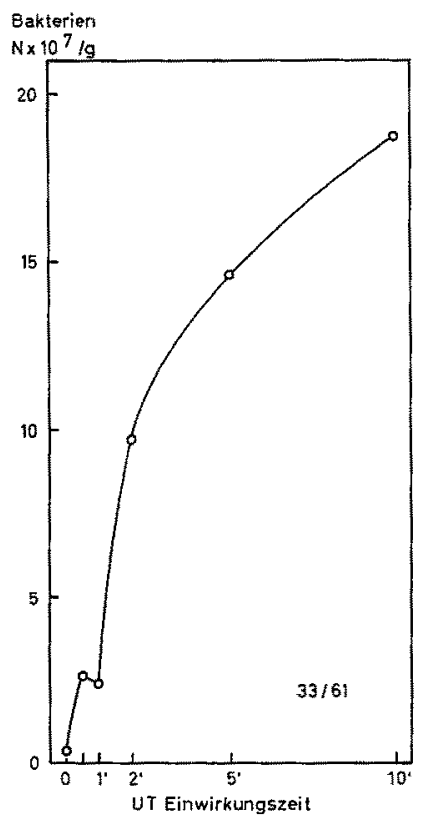

Abb. 6: Einfluß der Homogenisation auf die Bakterienzahl eines Strandsediments 
einer, wenn auch geringfügigen, Abtötung von Bakterien durch die Homogenisation gerechnet werden. Abbildung 3 zeigt die Aufteilung von Bakterienaggregaten in abgestorbenem Ceratienplankton. Die Bakterienzahl steigt nahezu auf das Doppelte, der

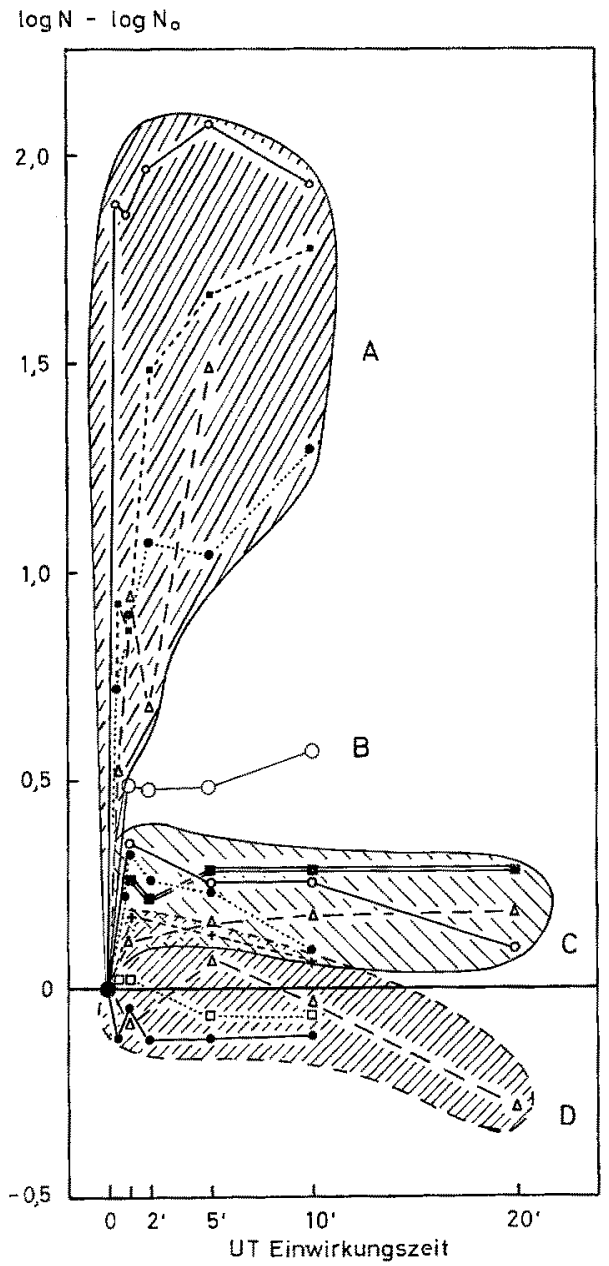

Abb. 7: Zusammenfassung des Einflusses der Homogenisation auf 13 verschiedene Proben marinen Ursprungs. $A$ Strandsedimente, reich an organischer Substanz beziehungsweise faulender Algen; $B$ Fischfleisch; $C$ küstenferne Sedimente bezichungsweise abgestorbenes Ceratienplankton; $D$ Seewasser

Maximalwert wird bereits nach ungefähr einer Minute Laufzeit erreicht. In Abbildung 4 ist die Aufteilung von Bakterienaggregaten einer Fischfleischprobe dargestellt. Die Ausgangszahl wird mehr als verdreifacht.

Weniger stark ist der Anstieg der Bakterienzahl, der nach Behandlung einer Sedimentprobe erhalten wird, die bei Tonne Hever I mit dem Bodengreifer entnommen worden war (Abb. 5). Es handelte sich hier um einen anaeroben sandigen Schlick 
mit Schlickeinsprengseln. Das Redoxpotential betrug $\mathrm{Eh}=-208 \mathrm{mV}$, der pH 7,55. Der Anstieg geht hier langsamer vor sich als in den anderen Proben.

Abbildung 6 ist ein Beispiel für ein strandnahes Sediment. Es handelt sich hierbei um einen anaeroben Sand aus der Nähe der Niedrigwassergrenze, der in Kontakt mit Wasser stand, das aus einer Ansammlung von in Fäulnis begriffenen Algen herauslief. Der Sand war grauschwarz gefärbt. Durch die Aufteilung mit Hilfe des Ultra-Turrax findet eine gewaltige Zunahme der nachweisbaren Bakterien statt. Die Kurve läßt erkennen, daß die Aufteilung selbst nach einer Laufzeit von 10 Minuten noch nicht völlig abgeschlossen ist.

Die eben besprochenen Kurven sind mit weiteren Versuchsreihen in Abbildung 7 zusammengefaßt. Um einen Vergleich zu ermöglichen, sind die Ergebnisse in einem semilogarithmischen Raster aufgetragen. $\mathrm{Da}$ auf der Ordinate für die Anzahl Bakterien der Ausdruck $\log \mathrm{N}$ minus $\log$ No gewählt wurde (vom jeweiligen Logarithmus der erhaltenen Zahl wurde der Logarithmus der Anzahl Bakterien der unbehandelten Probe abgezogen), beginnen sämtliche Versuchsreihen bei 0 .

Die 13 Versuchsreihen verteilen sich eindeutig in 4 Gruppen. Gruppe A umfaßt strandnahe Sedimente, die reich an organischer Substanz waren, bzw. verfaulende Algen vom Strandsaum. Es findet eine gewaltige Zunahme der Bakterienzahl statt. Gruppe B mit noch sehr starker Zunahme ist die behandelte Fischfleischprobe. Gruppe C enthält küstenferne Sedimente sowie die homogenisierte Ceratienplanktonprobe. Wir finden noch eine eindeutige Steigerung, die jedoch nicht mehr die Werte von Gruppe A und Kurve B erreichen. Gruppe D schließlich umfaßt Seewasserproben mit Werten, die gelegentlich über, häufig jedoch unter dem Ausgangswert liegen. Die in jeder Versuchsreihe erhaltene Maximalzahl ist in Tabelle 1 in Prozent des Ausgangswertes aufgeführt.

Tabelle 1

Maximalzahlen der Versuchsreihen in Prozent

\begin{tabular}{|c|c|}
\hline Versuchsnummer & $\begin{array}{l}\text { Bakterienzahl in \% } \\
\text { des Ausgangswertes }\end{array}$ \\
\hline $\begin{array}{c}\text { Gruppe A (Küsten-Se } \\
25 / 61 \\
32 / 61 \\
33 / 61 \\
36 / 61\end{array}$ & $\begin{array}{l}\text { te, Versuch } 32 / 61 \text { : verfaulende Algen vom Strandsaum) } \\
3080 \\
1930 \\
5886 \\
11863\end{array}$ \\
\hline $\begin{array}{c}\text { Gruppe B (Fischfleisch) } \\
66 / 63\end{array}$ & 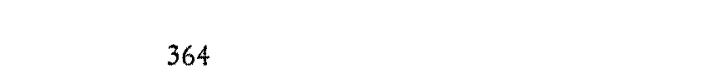 \\
\hline $\begin{array}{c}\text { Gruppe C (Küstenfern } \\
30 / 61 \\
50 / 61 \\
50 / 61 \\
47 / 61\end{array}$ & $\begin{array}{ll}\text { limente bzw. Ceratienplankton) } \\
212 & \text { (Sed. Otzumer Balje) } \\
157 & \text { (Sed. Hever I) } \\
227 & \text { (Sed. Hever III) } \\
195 & \text { (Ceratienplankton) }\end{array}$ \\
\hline $\begin{array}{c}\text { Gruppe D } \text { (Seewasserp } \\
24 / 61 \\
27 / 61 \\
59 / 61 \\
64 / 61\end{array}$ & $\begin{array}{r}105 \\
142 \\
75 \\
174\end{array}$ \\
\hline
\end{tabular}


Die Ergebnisse zeigen, daß die mit verwandtem Nährboden nachweisbaren Bakterien in den verschiedenen untersuchten marinen Proben stark unterschiedliche Aggregationen bildeten. Die durchschnittliche Größe betrug bei den Seewasserproben bis 1,7, bei den küstenfernen Sedimenten beziehungsweise dem abgestorbenen Ceratienplankton 1,6 bis 2,3, im Fleisch eines tiefgefrorenen Fisches 3,6 und in Küstensedimenten beziehungsweise faulenden Algen 19 bis 119. Während also durch Homogenisation in der Seewasserprobe keine nennenswerte Steigerung der Bakterienzahlen erreicht werden konnte, wurde bei Sedimenten des nährstoffreichen Typus, die in Strandnähe entnommen wurden, eine bis über 100 fach höhere Zahl erhalten.

Der Ultra-Turrax ist preiswert, sehr effektvoll und einfach zu handhaben. Ohne großen Aufwand kann mit diesem Gerät unter sterilen Bedingungen gearbeitet werden, wobei nur geringe Probemengen notwendig sind. Die Anwendung eines UltraTurrax bei der bakteriologischen Untersuchung nährstoffreicher Sedimente ist daher sehr zu empfehlen.

\section{ZUSAMMENFASSUNG}

1. Mit Hilfe eines Homogenisators der Type Ultra-Turrax Typ $18 / 2$ wurden Aufteilungen von Bakterienaggregaten in marinen Proben durchgeführt, da die verwandten Kulturverfahren nur in der Lage sind, räumlich voneinander getrennte Bakterien zu erfassen.

2. Um die Temperatur der Probe während der Laufzeit des Gerätes nicht in schädliche Bereiche ansteigen zu lassen, ist eine Kühlung durch ein Eis-Wassergemisch notwendig.

3. Bei Seewasserproben wurden stark wechselnde Ergebnisse erhalten, die teilweise geringfügig über, teilweise unter dem Ausgangswert lagen.

4. Bei küstenfernen Sedimenten wurde nach Homogenisation eine Steigerung der Keimzahl bis auf das 2,3fache erzielt.

5. Eine Fischfleischprobe (tiefgefrorener Hering) ergab bis zum 3,6fachen der Ausgangszahl.

6. Bei küstennahen Sedimenten, die reich an organischer Substanz waren beziehungsweise bei sich zersetzenden Algen, wurde durch die Behandlung mit dem UltraTurrax eine gewaltige Steigerung der mit dem Gußplattenverfahren erfaßbaren Bakterien erreicht. Sie betrug in 4 Versuchsreihen das 31-, 19-, 59- und 119fache der nicht homogenisierten Probe. Im Interesse einer vollständigeren Erfassung der Bakterien wird bei solchen Proben die Anwendung des Ultra-Turrax vorgeschlagen.

Frau E. Waltemath und Herrn H. H. Trekel, der auch die Zeichnungen anfertigte, bin ich für ihre gewissenhafte Mitarbeit zu großem Dank verpflichtet. 


\section{ZITIERTE LITERATUR}

Buck, J. D. \& Cleverdon, R. C., 1961. The effect of Tween 80 on the enumeration of marine bacteria by the spread and pour plate methods. Limnol. Oceanogr. 6, 42-44.

Fuchs, O., 1960. Über hochfrequente Stoffbehandlung. Chemikerzeitung - chem. Appar. 84, 809-814.

Jones, G. E. \& Jannasch, H. W., 1959. Aggregates of bacteria in sea water as determined by treatment with surface active agents. Limnol. Oceanogr. 4, 269-276.

Tron (o. Jahresangabe). Anwendung der Membranfilter-Methode in der Lebensmittel- und Getränkeindustrie. Membranfilter-Ges. Göttingen: Membranfilter-Inform. M 115.

WILLEMs, P., 1956. Mechanische Hochfrequenzgeräte und -verfahren in der chemischen Technik. Dechema-Monogr. 28, 173-190.

ZoBeLL, C. E., 1946. Marine microbiology; a monograph on hydrobacteriology. Chronica Botanica, Waltham, Mass., 240 pp. 